\title{
In vitro cultivation of Toxocara cati adult worms for production of eggs and evaluation of oviposition
}

\author{
M. ZIBAEI ${ }^{1,2}$, S. M. SADJJADI ${ }^{1, *}$, B. SARKARI ${ }^{2}$, A. ORYAN ${ }^{3}$, S. UGA ${ }^{4}$ \\ ${ }^{1}$ Department of Parasitology and Mycology, School of Medicine, Shiraz University of Medical Sciences, Shiraz, Iran, \\ *E-mail: smsadjjadi@sums.ac.ir $;{ }^{2}$ Department of Parasitology and Mycology, School of Medicine, Lorestan \\ University of Medical Sciences, Khorramabad, Iran; \\ ${ }^{3}$ Department of Pathology, School of Veterinary Medicine, Shiraz University, Shiraz, Iran; ${ }^{4}$ Department of \\ Parasitology, Faculty of Health Sciences, Kobe University Graduate School of Health Sciences, Kobe, Japan
}

\begin{abstract}
Summary
Toxocariasis is the clinical syndrome caused by infection of zoonotic roundworms of dogs (Toxocara canis) or cats (Toxocara cati). Current research on the immunology and pathology aspects of toxocariasis requires Toxocara second stage larvae and a ready source of excretorysecretory (ES) antigens. We cultured eleven pairs of both sexes of Toxocara cati adult worms maintained in RPMI 1640 medium in order to evaluate the amounts and duration of egg laying. At the first day and last day (day 19), the mean egg counts were 9300 and $250 \mathrm{eggs} / \mathrm{ml}$, respectively. These results showed that this culture method is very appropriate for collection of pure oviposited eggs and/or production of adult ES antigens of Toxocara cati that could be used for immunological diagnosis of toxocariasis.
\end{abstract}

Key words: Toxocara cati; cultivation; in vitro; Adult worms

\section{Introduction}

Toxocariasis is the clinical term applied to infection in the human host with either Toxocara canis ( $T$. canis) or Toxocara cati (T. cati) (Despommier, 2003). Infection can occur by ingestion of embryonated eggs from the soil or ingestion of larvae in uncooked meat of infected paratenic hosts such as chicken, cattle or sheep (Azizi et al., 2007). The prevalence of seropositivity varies from 2.6 to $86 \%$ in different parts of the world (Sadjjadi et al., 2000). Reports on Toxocara eggs contamination of soil samples from public areas in various parts of the world showed that the contamination rates ranged from 6 to $92 \%$ (Zibaei \& Uga, 2008).

Reports suggest that the spectrum of toxocaral disease goes

* corresponding author beyond the limits of Visceral Larva Migrans (VLM) and Ocular Larva Migrans (OLM) syndrome characterized by chronic weakness, abdominal pain various sign of allergy, mild hypereosinophilia (Magnaval et al., 1991). In the absence of parasitological evidence of infection, diagnosis of larval toxocariasis has relied mainly on immunological methods. Highly sensitive and specific serodiagnostic assays have been developed using Toxocara excretorysecretory (ES) antigens (Fife, 1971; de Savigny, 1975; Glickman et al., 1985). The large-scale product of this antigen is recovery of Toxocara spp. eggs which unembryonated passed in the feces of dogs and cats and/or in the uteri of female worms. Under optimal temperatures and humidity, these eggs develop into embryonated eggs, that is the principle of Toxocara ES antigens (Overgaauw, 1997). None of the reports from trails of in vitro cultivation of Toxocara cati adult worms were concerned with egg laying and collection of ES protein; although other helminths have been cultured successfully (Hoti et al., 1994; Chaitong et al., 2001; Walter, 1998; Alarcon de Noya et al., 1992; Hemphill et al., 2002). However, these data are important to evaluate the usefulness of an in vitro model for assemblage of ES antigens of adult worms for comparison with second stage larvae and also eggs laid.

The purposes of this study were to evaluate the duration of in vitro cultivation of $T$. cati adult worms and further to clarify the oviposition in these worms.

\section{Materials and Methods}

Recovery of adult worms

Toxocara cati adult worms were obtained by necropsy from stray cats in different parts of Shiraz, Iran. Female worms were isolated and rinsed first in distilled water and then phosphate buffered saline $(\mathrm{pH}$ 7.2) to remove adherent faecal material. 


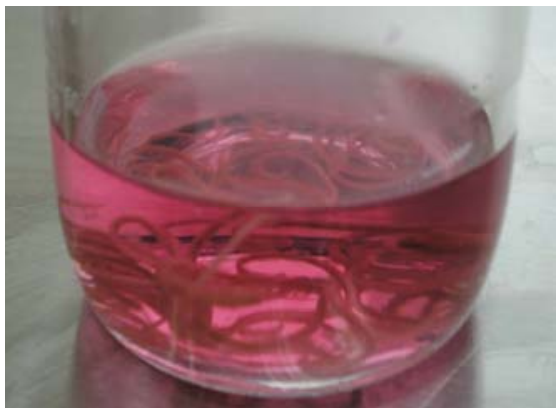

Fig. 1: Toxocara cati adult worms were cultured in RPMI 1640 medium

In vitro culture

After three to four washes with Ringer. 1/4 Ringer's solution was prepared by dissolving 1 tablet including Ammonium chloride 0.4; sodium hydrogencarbonate 0.005; calcium chloride-2-hydrate 0.040; potassium chloride 0.0525 ; sodium chloride 1.125 , (Merck, Germany) in $500 \mathrm{ml}$ neutral deionized water and sterilized in the autoclave $\left(15 \mathrm{~min}\right.$ at $\left.121^{\circ} \mathrm{C}\right), \mathrm{pH}: 6.9 \pm 0.1$ at $25^{\circ} \mathrm{C}$. A total of 11 male/female pairs were separated and transferred to $20 \mathrm{ml}$ of sterile RPMI 1640 (Gibco BRL, Netherlands) containing Penicillin/Streptomycin (at 100 $\mathrm{U} / 100 \mu \mathrm{g} / \mathrm{ml}$ ) (Sigma - Aldrich Chemie GmbH, Germany), sodium bicarbonate, HEPES buffer (Merck, Germany), Amphotericin B $(50 \mu \mathrm{g} / \mathrm{ml})$ (Bristol-Myers Squibb, Netherlands) and $1 \%$ glucose. The medium was maintained at $37{ }^{\circ} \mathrm{C}$ and $5 \%$ carbon dioxide and $95 \%$ nitrogen in a $\mathrm{CO}_{2}$ incubator (Zibaei et al., 2007).

\section{Egg recovery}

The medium was changed twice a day and then daily by tilting the culture flask to allow the adult worms and eggs to sediment and then aspirating of media. The flasks were then replaced with fresh medium. The sediment (eggs were released from females) was centrifuged at $1500 \mathrm{~g}$ for 3 minutes for recovery of eggs. The eggs were examined under an inverted microscope and/or stereoscope and counted every 24 hours for 19 days.

\section{Results}

Toxocara cati adult worms were cultured in RPMI 1640 for 19 days (Fig. 1). Most of the eggs were recovered in 24 hours with $9300 \mathrm{eggs} / \mathrm{ml}$ and after 48 hours, the numbers of eggs were 9000 -eggs $/ \mathrm{ml}$. Thus after 19 days the numbers of eggs were decreased dramatically and only a few eggs were recovered (Fig. 2). Changes in the developmental stage of T.cati eggs recovered from RPMI 1640 medium are shown in Table 1.

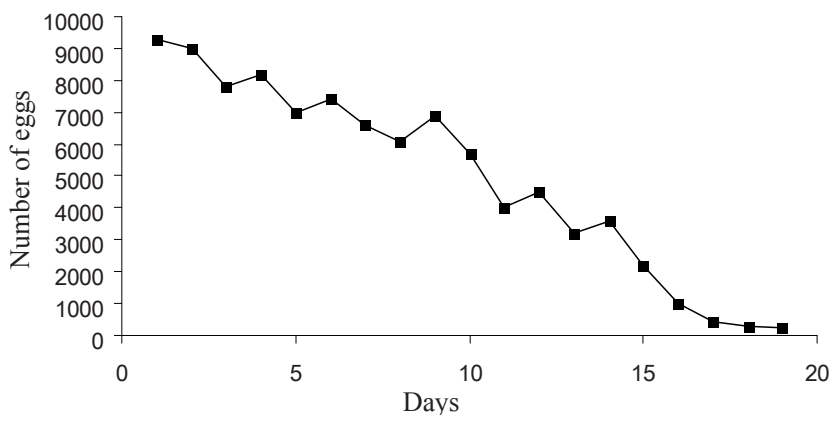

Fig. 2: Daily recovery of Toxocara cati eggs from 11 pair adult worms/20ml RPMI 1640 medium within 19 days

\section{Discussion}

There have been numerous reports on the development of an in vitro culture technique for helminths to obtain ES products from parasites (Healer et al., 1991), to screen the effects of anthelmintics (Rew et al., 1986), to evaluate nutritional equipments of larvae and adult worm (Urban et al., 1984), to determine biochemical changes associated with parasite development (Gamble \& Mansfield, 1996) and for immunodiagnosis and also to evaluate infectivity of egg (Baek et al., 1998).

An in vitro maintenance of adult stage parasite in some of helminthes for experimental investigation requires a serial passage in susceptible laboratory animal, which are not only an expensive work but also a time consuming one.

In some of parasitic diseases including toxocariasis, current diagnosis is serodiagnostic testes that measured total immunoglobulin against ES proteins recovered from in vitro cultured infective larvae (de Savigny, 1975). The production of this antigen need eggs that after full embryonation hatch in vitro according to the methods have reported (Villanueva \& Kozek, 1992).

Up until now, have been different reports on the development of in vitro culture techniques for second stage larvae $T$. cati to obtain ES products, but no reports from in vitro cultivation of $T$. cati adult worms. RodriguezCaballero et al. (2007) have compared the population size of fertilized eggs from Toxocara canis female worms kept in culture and those obtained using a uterus excision method. In vitro culture may be useful in order to

Table 1. Situational changes of Toxocara cati eggs in medium

\begin{tabular}{ccccc}
\hline Days & Total No. of eggs & \multicolumn{2}{c}{ No. of eggs } & Embryonation \\
\cline { 3 - 4 } & & unembryonated & embryonated & $(\%)$ \\
\hline $0-5$ & 41292 & 41172 & 120 & 0.3 \\
$6-10$ & 32700 & 32338 & 362 & 1.1 \\
$11-15$ & 17580 & 17135 & 445 & 2.5 \\
$16-19$ & 1973 & 1792 & 181 & 9.2 \\
\hline Total & 93545 & 92437 & 1108 & 13.1 \\
\hline
\end{tabular}


harvesting T. cati adult worm ES antigen for comparison to $\mathrm{L}_{2}$ stage and also eggs for future study. However, we did not determine the survival period of adult worms in this medium.

The present study was designed to use a simple in vitro culture system for maintenance of $T$. cati adult worms as well as the technique for extending the collection of eggs and ES antigens. The method described in this study is simple to perform and will be available commercially. We feel it may serve as a base for future Toxocara cati research projects.

\section{Acknowledgment}

We wish to express our gratitude to N. Tanideh, S. Azizi, A. Roeintan, A. Baziari, S. Kazemian and B. Farhangmehr for their help in the field and technical assistance.

\section{References}

Alarcon De Noya, B., Torres, J. R., Noya, O. (1992): Maintenance of Sparganum proliferum in vitro and in experimental animals. Int. J. Parasitol., 22: 835 - 838

AZIZI, S., Oryan, A., SAdJjAdi, S. M., Zibaei, M. (2007): Histopathologic change and larval recovery of Toxocara cati in experimentally infected chickens. Parasitol. Res., 102: $47-52$

BAEK, B. K., Islam, M. K., KIM, J. H. (1998): Viability of eggs, filariform larvae and adults of Strongyloides venezuelensis (Nematoda: Strongyloidea) maintained in vitro. Korean J. Parasitol., 36: 99-107

Chitong, U., Sukontason, K., Boonsriwong, N., Sukontason, K. L., PianguaI, S. (2001): In vitro development of Haplorchis taichui (Trematoda: Heterophyidae). Southeast Asian J. Trop. Med. Public Health, 32: 31 $-35$

DE SAVIGNY, D. H. (1975): In vitro maintenance of Toxocara canis larvae and a simple method for the production of Toxocara ES antigen for use in serodiagnostic tests for Visceral Larvae Migrans. J. Parasitol., 61: 781 - 78

DesPommier, D. (2003): Toxocariasis: clinical aspect, epidemiology, medical ecology, and molecular aspects. Clin. Microbiol. Rev., 16: 265 - 272

FIFE, E. H. (1971): Advances in methodology for immunodiagnosis of parasitic disease. Exp. Parasitol., 30: $132-$ 163

GAMBLE, H. R., MANSFIELD, L. S. (1996): Characterization of excretory-secretory products from larval stages of Haemonchus contortus cultured in vitro. Vet. Parasitol., 62: $291-305$

Glickman, L. T., Grieve, R. B., LAuria, S. S., Jones, D. L. (1985): Serodiagnosis of ocular toxocariasis: A comparison of two antigens. J. Clin. Pathol., 38: $103-107$
Healer, J., Ashall, F., Maizels, R. M. (1991): Characterization of proteolytic enzymes from larval and adult Nippostrongylus brasiliensis. Parasitology, 103: 305 - 314 Hemphill, A., Stettler, M., WalKer, M., Siles-Lucas, M., FinK, R., GotTsteIn, B. (2002): Culture of Echinococcus multilocularis metacestodes: an alternative to animal use. Trends Parasitol., 18: 445 - 449

Hoti, S. L., Gros, A. M., Paily, K. P., Manonmani, A. M., MAry, K. A., Blaraman, K. (1994): In vitro cultivation of third stage larvae of Wuchereria bancrofti to fourth stage: influence of some physicochemical factors. Southeast Asian J. Trop. Med. Public Health, 25: $278-$ 283

Magnaval, J. F., Faber, R., Maurieres, P., Charlet, J. P., DE LARRAD, B. (1991): Application of the Western blotting procedure for the immunodiagnosis of human toxocariasis. Parasitol. Res., 77: $697-702$

Overganuw, P. A. M. (1997): Aspects of Toxocara epidemiology: Toxocariasis in dogs and cats. Crit. Rev. Microbial., 23: $233-251$

Page, A. P., Maizels, R. M. (1992): Biosynthesis and glycosylation of serine/ threonine-rich secreted proteins from Toxocara canis larvae. Parasitology, 105: 297 - 308 Rew, R. S., Urban, J. F. JR., Douvers, F. W. (1986): Screen for anthelmintics, using larvae of Ascaris suum. Am. J. Vet. Res., 47: $869-873$

Rodriguez-CABAllero, A. R., LUnA-OChOA, R. I., Ponce-Macotela, M., Peralata-Albarca, G. E., MARTinez-Gordillo, M. N. (2007): A simple and inexpensive in vitro method for retrieving fertilized Toxocara canis eggs. Parasitol. Res., 101: $829-832$

Sadjuadi, S. M., KhosRaVi, M., Mehrabani, D., Oryan, A. (2000): Seroprevalence of Toxocara infection in School Children in Shiraz, Southern Iran. J. Trop. Pediatrics., 46: $327-330$

Urban, J. F. JR., Douvers, F. W., Xu, S. (1984): Culture requirements of Ascaris suum larvae using a stationary multi-well system: increased survival, development and growth with cholesterol. Vet. Parasitol., 14: 33 - 42

Villanueva, E. J., KozeK, W. J. (1992): A method for the recovery of infective larvae from Toxocara canis eggs for collection of larval secretory/excretory products. Trop. Vet. Med., 653: $211-216$

WALTER, R. D. (1998): In vitro maintenance of Onchocerca volvulus for harvest of excretory and secretory products. Trop. Med. Parasitol., 39: 448 - 449

ZiBAeI, M., UGA, S. (2008): Contamination by Toxocara spp. eggs in sandpits in Kobe, Japan. Journal of Environmental Control Technique, 26: 32 - 37

Zibaei, M., Sadjuadi, S. M., Jahadi Hosseini, S. H., SARKARI, B. (2007): A method for accelerating the maturation of Toxocara cati eggs. Iranian. J. Parasitol. , 2: $39-$ 42

ACCEPTED JANUARY 26, 2009 